\title{
Basic Gene Discretization-Model using Correlation Clustering for Distributed DNA Databases
}

\author{
Dr.Vijay Arputharaj J \\ School of Science \& Information Technology (SSIT), Skyline University, Nigeria \\ Email: vijay@sun.edu.ng,phdvij@gmail.com \\ Ms.Pushpa Rega Ganesan \\ Department of Software Engineering, Jigjiga University, Ethiopia \\ Email: pushparega994@gmail.com \\ Mr.Ponsuresh Manoharan \\ Department of Information Technology, Jigjiga University, Ethiopia \\ Email: ponsuresh.techie@gmail.com \\ Ms.P.Supraja \\ Assistant Professor, Hindusthan College of Arts and Science, Coimbatore, India \\ Email: suprajabalakrishnan@gmail.com
}

\begin{abstract}
Gene is a basic component of DNA located in the nucleus of Human cell. Currently data mining technique has huge impact in fields of human genetic science and gene sequence data analysis. Gene sequence analysis is a method of subjecting DNA sequence to systematic methods in order to know the genes character, configuration, nature and characteristics. $\mathrm{CBC}$ and $\mathrm{MNBC}$ applied to gene sequence data analysis, aims to segregate diseased diabetic genes from a vast stream of DNA gene sequence elements present in group of copious statistical data. This techniques attempts to approve, determine methods and tools for analyzing diseased gene sequences. It also helps in classification and interpretation of results accurately and meaningfully. This study is a combination of supervised and unsupervised machine learning technique for data analysis. The clustering is done by CBC whereas classification done by MNBC techniques. It recognizes gene expressions by framing association rules in accordance with support measure and confidence measure on the input data set.It will extract and filter required data into clusters based on CBC technique thereby drafting association rules. These are then applied on testing dataset to filter required (diseased) gene sequences. Finally MLRC algorithm is applied as classification algorithm to identify class labels of test genes sequences in a big dataset. In medical diagnosis gene data mining techniques through gene discretization models helps to identify various associations between the DNA genes based progressions and inconsistency in disease infections transformations. Above all it overcomes the limitation of existing Support Vector Machine Classification technology which incurs high computational cost and increased iterations
\end{abstract}

Keywords - Data mining, Data Analysis, DNA Gene, Gene Sequence, Vector Machine Classification

Date of Submission: Apr 19, 2020

Date of Acceptance: May 08, 2020

\section{INTRODUCTION}

The first phase of research work is associated with the genetic material discretization of basic DNA genomic elements. In the field of biomedical science, health disorders and their characteristics have a huge relationship with the gene expressions. The basic elements of genetic material discretization deal with Introns and Exons[1]. DNA is made up of one percent protein coding called genes, the rest of DNA are non coded genes. These coded DNA genes are also known as 'exons'. The non coded genetic elements are described as 'introns'. The figure illustrates the basic genetic materials such as introns and exons in nucleotide sequence of a small part of gene. It shows that the short gene surrounds with single introns and have multiple exons shown. (Figure1.1)

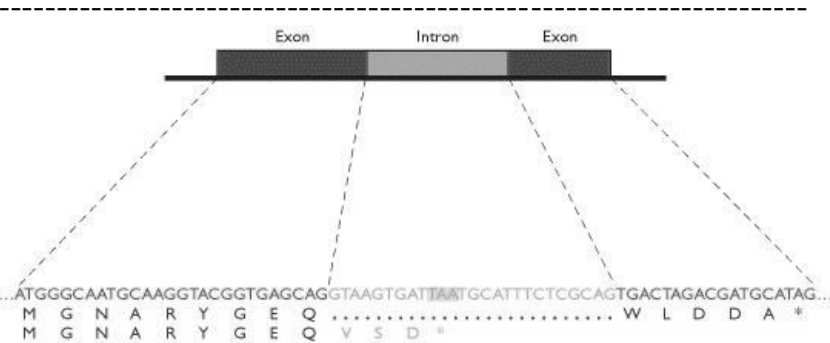

Figure 1.1 Introns and Exons in Nucleotide Sequence

The data mining technique has a huge impact and application in human genetics and gene sequence data analysis. In this proposed study data mining and DNA sequencing has its own problem definitions and objectives. For instance, to discover the advancements in genomic combination which reveals basic DNA genetic elements and other mutation elements, also to discover the natural merits of DNA genetic elements with regard to distribution of introns and exons, gene structures and active protein sites, etc. 
Introns- the word introns have come from intra-genic region. This intron is an interior region of genetic element. The introns refer to both the sequence of RNA transcription region and DNA sequence region. Introns are found in human genetic elements and in other creatures. Many viruses also contain introns in it. These introns may be a major part of gene and occupies a wide range over it. (Figure 1.2)

4 different classes of introns include:

1. Introns in nuclear protein genes.

2. Introns in nuclear and transferred RNA genes.

3. Self- splicing first group non coded introns

4. Self- splicing second group of non coded introns

5. Third group of non coded introns, proposed to be fifth similar to group-2

Exons have notable function but it founds a part of genomic sequence elements. It is discovered that functional nuclear exons can adopt sequence that is primary for the expression of the gene on the exons reside.
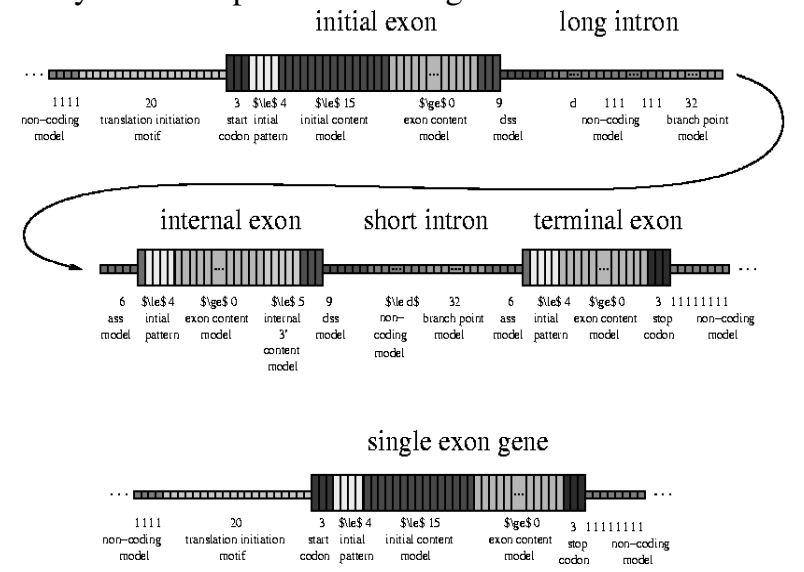

Figure 1.2 Detailed Introns and Exons in Nucleotide Sequence

This study introduces a proposed model as discretization of basic genetic material like introns and exons using supervised and unsupervised machine learning techniques such as CBC- one of the clustering type correlation clustering, naïve Bayesian classification (modified) and logistic regression (modified) for data analysis[2] of basic genomic elements. The proposed technology correlation based clustering creates the gene clusters followed by drafting different association rules which are applied on testing data with calculating support value and calculated confidence value to sort out required genetic elements. Finally, in order to discover set of labels the logistic regression (modified) classification is been used. The proposed technology will overcome the above increased iteration issues. It is also cost effective compared to support vector machine classification method which is in existing practice.

\section{REVIEW OF EXISTING TECHNOLOGY- SVM CLASSIFICATION MODEL}

The existing approach deals with microarray data classification models associated with SVM classification model[3] which applied as organized machine learning approach to facilitate the class model data from a genomic data. In this existing study, it uses the labeled gene expression samples. The labeled gene expressions classified by a classifier model. This classifier classifies the above samples into predefined parameters specified.

\section{SVM- SUPPORT VECTOR CLASSIFICATION:}

The SVM machine has an extension of another SVCsupport vector classification. This is also an unsupervised approach. This unsupervised algorithm generates kernel functions. This is one of the very important gene mining approaches. The following are varies extension SVM's which are in current practice.

\section{MULTICLASS SVM: (Multi class to single optimization) \\ The main goal of multiclass SVM is to initiate} different labels to the data instantaneously by using SVM. The labels are taken from the set of elements here; here the specified elements belong to gene array. This multiclass SVM approaches reducing the multi class problem to multiple binary classifications.

The following are the steps followed for above classification:

- Generation of binary classifier: this differentiates labels from other set of labels and other class labels.

- Error correction: error - correction output code for SVM.

\section{STRUCTRED SVM:}

This is also an extension of support vector machine learning algorithm. This method extends and generalizes the SVM algorithm. This is a best example to support regression as well as binary multi-task classification. This structured supports vector machines provide structured output labels.

\section{TRANSDUCTIVE SVM:}

Transduction Support vector machines are an extended machine of support vector machines. In this methods, the transduction principle is being followed and the data is been labeled using semi-supervised learning approach.

\section{BAYESIAN SVM:}

The Bayesian SVM is an approach that provides SVM method in a graphical model. This extended application of support vector machine to Bayesian SVM has numerous advantages such as feature modeling, parameter feature tuning etc.

\section{SUPPORT VECTOR REGRESSION-SVR:}

In 1996, the new extension of SVM as Support vector machine for regression is been discovered by Vladimir $\mathrm{N}$ Vapnik and his colleagues [4]. The SVM for regression is also known as SVR-Support vector regression. This technique evolving from SVM classification is based only on the subsets of trimming data. The cost value task constructs the SVM. This did not consider the trimming value point. The SVM model formed by SVR, it may work based on training data's subsets. 
Following Figure 2.1 shows the SVR prediction with different thresholds [4]. In this figure it generates different data points to data clusters as shown

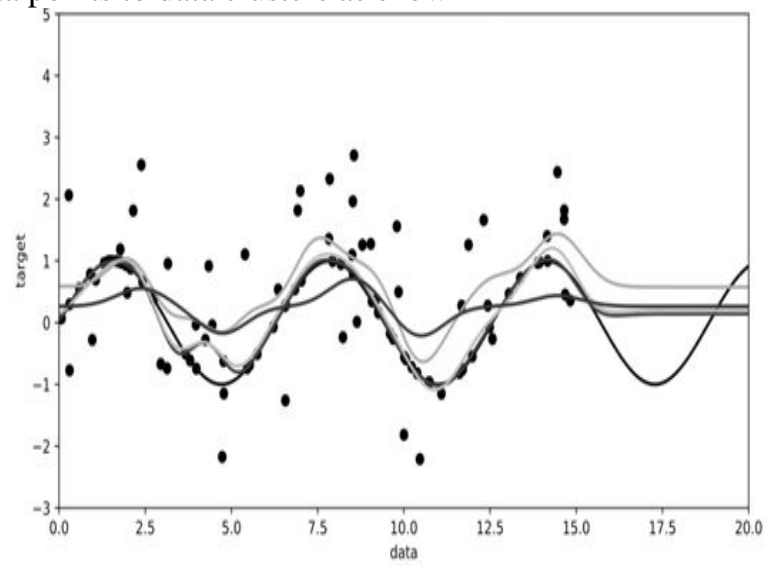

Figure 2.1 Support Vector Regression- Predictions with Different Thresholds

\section{IMPLEMENTATION:}

Before the SVM approach there were some difficulties identified from the existing approaches. The important issue that has been overcome from the existing approach includes the identification of informative gene sequences. Informative genes are the qualified genes. All other genes, other than informative genes are called as noise genes in the dataset. The informative genes and noise genes are the base for better training time and accuracy. In order to have better training time and accuracy, Sanz et al (2002) has proposed Reduced SVM method based on RFE(Recursive feature elimination)[5].

The approach based on SVM classification gets gene samples with labels initially. Then it generates a SVM classifier model. The classifier model is used to classify samples into pre defined specified parameters. In this approach SVM method is essential for micro-array data. The SVM works better and high dimensional data. This also helps in removing noisy data. The schematic diagram below represents the developed existing system. (Fig 2.2),

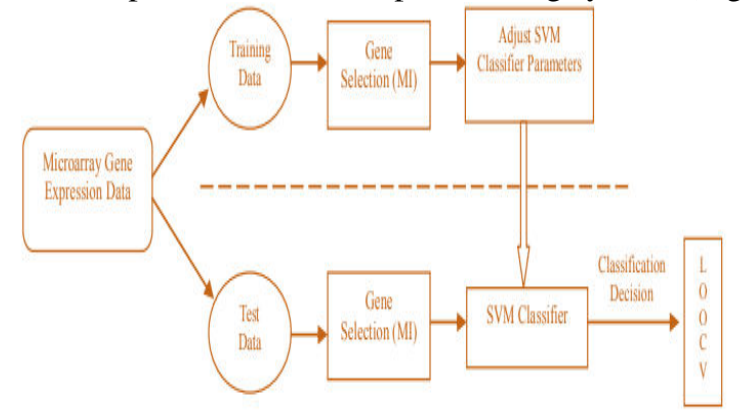

Figure 2.2. Representation of existing flow of SVM

Microarray genomic expression: This initializes microarray genomic expression with a gene sequence dataset.

Training Dataset: A training dataset consists of microarray gene expression dataset with examples used for decease prediction in specified parameters that is used to study and fit the parameters such as gene influence, disease type, etc. Most attempts to explore towards data to be trained intended for pragmatic association ships tend to over fit information, which means it will recognize, utilize evident associations with trained information data it didn't keep commonly.

Testing Dataset: A test dataset was an autonomous dataset unlike the previous dataset mentioned, but test dataset also tracks similar prospect of distribution as the training dataset. An improved data with proper training dataset contrasting to test data typically fits to over fitting of data.

Gene Selection: This sequence analyze to select the different micro-array genes for prediction

Adjust SVM classifier: This is a temporary class to adjust SVM classifier in several cases.

SVM Classifier: The classifier model is used to classify samples into pre defined specified parameters. In this approach SVM method is essential for micro-array data. The SVM works better and high dimensional data. This also helps in removing noisy data.

Logical regression: LR-Logistic regression is a process of forecasting the chances to be a gene characteristic, among ideals of autonomous variables.

\section{Simulation Results:}

The SVM classifiers for micro-array gene expression data among genetic expression information, the SVM have a capacity to differentiation between the subsets and non subsets of the given process oriented class. Leave One-Out is a cross validation technique which was analyzed to generalize and compute generated classifier model. By adopting this method we can avail data to the maximum extend, thereby avoiding the problems of random selection task. [6]

Table 2.1 Consequences of Gene sequence Experiments in SVM [6]

\begin{tabular}{|c|c|c|c|c|c|c|c|}
\hline $\begin{array}{l}\text { Type of } \\
\text { classifica } \\
\text { tion }\end{array}$ & $\begin{array}{l}\text { gene } \\
\text { sets }\end{array}$ & $\begin{array}{l}{ }^{\prime} \mathrm{T} \\
\mathrm{P}^{\prime}\end{array}$ & $\begin{array}{l}\mathrm{T} \\
\mathrm{T} \\
\mathrm{N}\end{array}$ & $\begin{array}{l}\text { 'F } \\
\text { P' }\end{array}$ & $\begin{array}{l}{ }^{\mathrm{C}} \mathrm{F} \\
\mathrm{N}\end{array}$ & $\begin{array}{l}\text { Correct } \\
\%\end{array}$ & $\begin{array}{l}\text { Fault } \\
\%\end{array}$ \\
\hline KNN & $\begin{array}{l}493, \\
1772\end{array}$ & 3 & $\begin{array}{l}1 \\
6\end{array}$ & 4 & 8 & $61 \%$ & $39 \%$ \\
\hline ANN & $\begin{array}{l}\& \\
1582\end{array}$ & 8 & 1 & 9 & 2 & $61 \%$ & $39 \%$ \\
\hline
\end{tabular}




\begin{tabular}{|c|c|c|c|c|c|c|c|c|}
\hline $\begin{array}{l}\text { SVM } \\
\text { Linear }\end{array}$ & \multirow[t]{4}{*}{)-3} & 3 & 2 & 0 & 9 & $74 \%$ & $26 \%$ & $\begin{array}{l}\text { III. OVERVIEW OF BASIC GENETIC } \\
\text { MATERIAL DISCRETIZATION MODEL }\end{array}$ \\
\hline $\begin{array}{l}\text { SVM } \\
\text { RBF }\end{array}$ & & 0 & 2 & 0 & 11 & $64 \%$ & $36 \%$ & \multirow{3}{*}{$\begin{array}{l}\text { Components of Genetic Material Discretization } \\
\text { The main components of genetic material are Coding } \\
\text { tole and Non-coding role of genes. A coding DNA is a } \\
\text { gene sequence of DNA. The Coding DNA codes for } \\
\text { protein in gene sequence. It is also known as Exons. The } \\
\text { Non-coding DNA sequences are also called as Introns. } \\
\text { This is one of the mechanisms of an individual's DNA } \\
\text { \$ene sequence that can't code protein\& mutation } \\
\text { \$equence. (Figure 3.1) }\end{array}$} \\
\hline $\begin{array}{l}\text { SVM } \\
\text { Quad }\end{array}$ & & 3 & 9 & 11 & 8 & $39 \%$ & $61 \%$ & \\
\hline $\begin{array}{l}\text { SVM } \\
\text { Poly }\end{array}$ & & 3 & 1 & 3 & 8 & $65 \%$ & $35 \%$ & \\
\hline
\end{tabular}

The SVM's- Support Vector Machines were robust and appropriate with data analysis classifiers. The classifier works as wide pattern of genetic data expression. This works better from microarray data[7]. SVM classifiers very easily covenant to huge amount of feature elements specified and little amount of distinct pattern specified in the samples. The issue in production with different features in enormous amount is removed through acquiring characteristic subdivision of specified SVM classification model .Informative genes are identified using mutual information of a classifier between genes, during a gene selection method. MI process has its impact over classification performance by SVM during the gene selection processes .Highest accuracy during classification of specific parameter was possible when SVM was with linear kernel.

Advantages of SVM Classifier:

- Support Vector Machines are performing better when the features is pretty large in number.

- Support Vector Machines work successfully even if the numbers of samples are lesser than the number of features. That means it works in higher features than datasamples.

- Other than linear informative datasets may be classified by applying SVM's. This is customized hyper planes constructed using kernel-tricks.

- These SVMs are a dynamic and strong form to work out various feature prediction issues.

Disadvantages of SVM Classifier:

- SVM's acquiring greater number of samples so it begins with reduced performance.

- SVM performs better simplification performance but it may be tremendously time-consuming with performance of test data.

- Support Vector Machine has elevated complication in algorithmic performance.

- SVMs have wide necessities towards the memory this led into increased cost.

- The very important drawbacks of SVM Machines are preferences of kernel elements. The incorrect preferences kernel elements may direct to boost in fault rate.
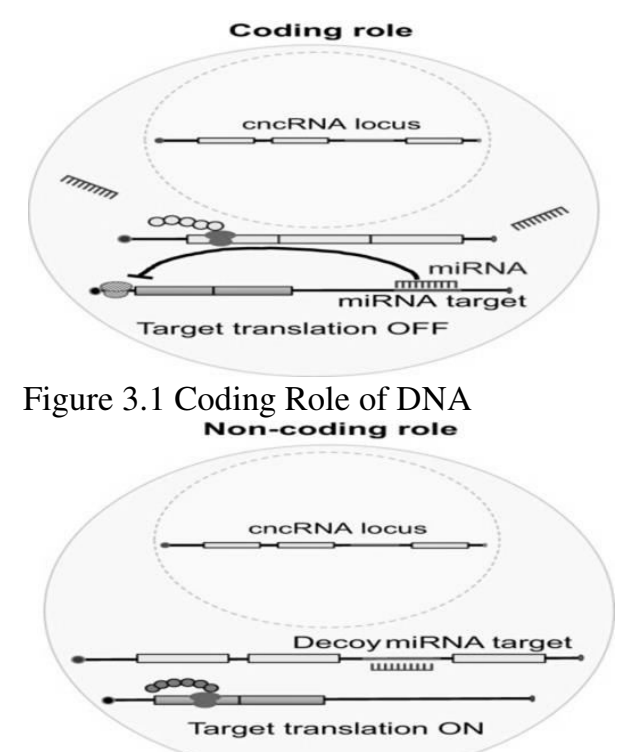

Figure 3.2 Non-Coding Role of DNA

Database IE- This database concerns with genetic material discretization of Introns and Exons. The data was provided by Gene Bank- Splice Dataset, it has several numbers of attributes and few targeted attribute. The complete data element contains 1000 instance as samples.

Dataset- Training purpose: A training dataset consists of splice gene bank dataset with examples used for gene discretization learning that is used to study and fit the parameters such as gene influence, disease, age etc. Most attempts to finding in the data which has been trained for pragmatic association-ships are likely to over fit information, which means it may recognize also utilize obvious association-ships of the data has been trained it don't clutch generally.

Dataset-Testing purpose: Test datasets have an autonomous dataset unlike the dataset used for training purpose, but test dataset also tracks similar prospect of sharing as the previous dataset mentioned. An improved strength of a dataset which has been trained as contrasting with the dataset has been trained normally link to over fitting of data.

Clustering genes: Clustering is defined as a process of grouping gene data elements with the group with regard to its own resemblance. 
Clustering technique (Correlation Clustering): Provides a method for clustering a set of genes into the optimum number of gene clusters without specifying that number in advance.

Regression Technique (Modified LR): Modified LR is used for prediction a gene characteristic, this also sort out if the characteristic is not similar with the above, in that there are some autonomous variables, those variables also considered as predictors to predict the gene characteristic. Based on the predictors and the rules to set the characteristic of gene sequences, it generates the suited genetic elements and the elements which not suited with the genetic sequence elements.

Strengths and Limitations of Proposed Algorithm

The most important strength in the gene mining algorithms is intrinsically parallel. Better computational and experimental methods have been implemented to analyze genome sequences by the proposed algorithms. Most of other algorithms naturally they are serial. The serial algorithms may discover elucidation to specific setbacks in particular way on an instance, also if solved problem which find out and return to an optimal solution. This cannot be solved but it will discard the issues and start afresh. The proposed technique manages very large datasets such as big data, cloud data and with a lot of noise. Our technique performs well in multi-class predictions. The generated output can be interpreted as probability.

The one of the few limitations of the proposed technology is very hardly handling categorical features. The binary features are not encouraged in this proposed algorithm. but most of genomic data incorporated with text categorical features. So the proposed technology can easily overcome this limitation. If a categorical variable in an algorithms have a category in the test dataset which was not available in the training dataset, then the category gets dispensed as zero probability and the proposed model is not capable of predicting the result. The solution for the above problem will be using Laplace smoothing technique[8].

\section{Flow of Research}

Different methods for correlation based clustering are available the relationship to different types of clusters are established using definite patterns. This research over indenture during the evolution of genetic based algorithm with apposite protection surfaces in DNA genetic gene databases which was called Splice Dataset[9].

The flow of this research contains different elements (Figure 3.3).

- The first element deals with training process and testing process with its own datasets contains:

Dataset- Training Process,

Dataset- Testing Process,

- The next process of flow of the research enhanced through different rules for associating the relation are performed, some of rule for associating here are:
To find support

To find confidence

To find mean

To do sequence pruning,

- The next process continues with supervised learning process clustering, the already generated data with applied association rules are continued with clustering (CBC). This is exposed in the Fig 3.3.

- The proposed technique has important tasks like Clustering (Correlation), LR Classification (Modified) as the part of genetic sequence data discovery process[10]

It primarily deals with the training dataset contains various different types of gene expressions were found as $\mathrm{i} / \mathrm{p}$ dataset with gene discretization model to be accepted. These i/p dataset have different genomic sequential elements and different labels for its grouped classes. As mentioned before, the rules for association by manipulating measurement of support value and finding the value of confidence have filtered above different genomic discretized elements noticeably.

The CBC-Clustering process was applied in generation of the different clusters in gene discretization model environment. Then, the process of testing elements was initiated by providing dataset which to be tested as an input of the system, and continued with similar process carried with the before mentioned dataset-training. Except the sequence pruning process all the other works are carried and at last $\mathrm{CBC}$ and $\mathrm{LR}$ (modified) is been applied. These two techniques are common process to both the dataset- training and dataset-testing.

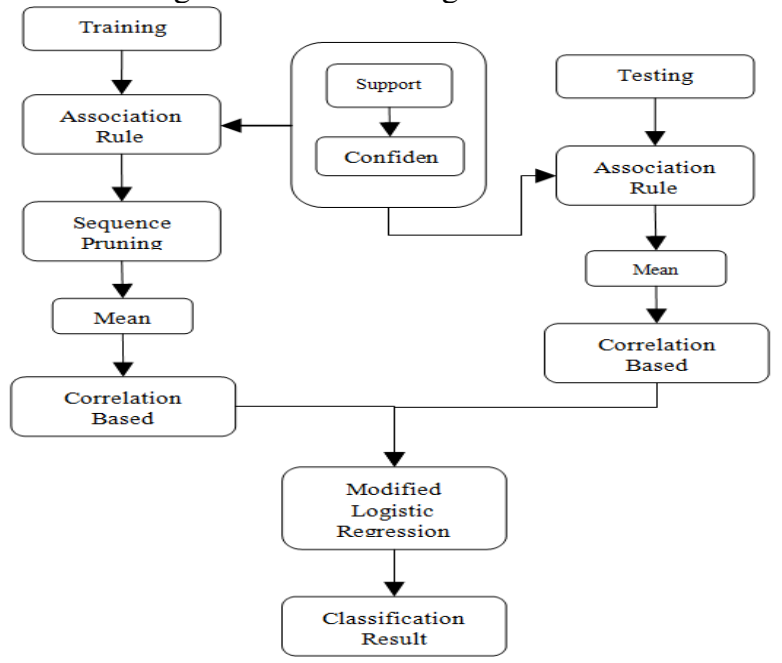

Figure 3.3 Research Flow of Proposed Study

\section{MATERIALS AND METHODS}

Gene mining with big data analytics: The first important material used was data mining technique. It was a data inspection process to contrivance information in the Gene Datasets in the DNA Databases. This is a very interesting inter discipline way of in general area of computer field combines bio medical field. Data mining contains different computations and calculations. This is an improvement of prototypes in enormous information DNA datasets connecting information systems at the association 
relationships of reproductions, in sympathetic methodology, knowledge discovery related information database schemes. The primary objective of genomic mining was generating data related to gene sequence in the given sequence from a large dataset of DNA database. The secondary objective is to modernize the generated data to intense the understandable establishment for supporting idea

\section{Application of association rules:}

It is one of machine learning method applies various rules for associations and to find out exciting genomic relationships among different reference variables in huge genomic big data.

Application of association rules[11] are proposing in identification of dynamic rules revealed in genomic big data by applying few actions in associating relations.

The rules applied for associating the relations mentioned below,

Where $\mathrm{G}=\{\mathrm{g} 1, \mathrm{~g} 2, \mathrm{~g} 3 \ldots \ldots \ldots . \mathrm{gn}\}$, in this $\mathrm{g}$ is a set of genomic elements, $\mathrm{n}$ is a length of the set.

Let $\mathrm{H}=\{\mathrm{h} 1, \mathrm{~h} 2, \mathrm{~h} 3 \ldots \ldots \ldots . . . \mathrm{hm}\}$ it is a set of genetic sequential elements such as mutation genes from the gene sequence database.

Each DNA sequence $H$ has an exclusive operational identification and has subsets of genes in $\mathrm{G}$.

These rules are specified by an inference of an element

$\mathrm{X} \& \mathrm{Y}$, Let $\mathrm{X}$ is 'antecedent', $\mathrm{Y}$ is 'consequent'

$\mathrm{X}=>\mathrm{Y}, \quad$ as derived $\mathrm{X}, \mathrm{Y} \subset \mathrm{G}$

This rule was defined only between a diseased diabetic gene sequence and a single gene

$\mathrm{X}=>\mathrm{ij}$ for $\mathrm{ij} € \mathrm{G}$

Each rule has collected by dissimilar group of genomic elements; these are genesets,

\section{Support Rule}

This rule is an indication to find out how frequently the coded gene proteins appearing from genomic big data. Support value calculated for ' $\mathrm{X}$ ' genes through ' $\mathrm{T}$ ' are identified as a ratio of diseased diabetic gene sequence $h$ in that dataset has gene for protein sequence.

$$
\operatorname{Sup}(X)=|\{h € H ; X \subset h\}|
$$$$
|\mathrm{H}|
$$

\section{Confidence Rule}

This rule is an indication to investigate how often the rules for association framed have established as true. The value of confidence measurement for rule can be measured by $\mathrm{X}=>\mathrm{Y}$ among the group of diseased or mutation gene sequence $\mathrm{H}$ is classified as ratio of diseased diabetic gene sequence that may have $\mathrm{X} \& \mathrm{Y}$.
Rule for confidence can be represented as

$\operatorname{Con}(X=>Y)=\sup \left(X U^{\prime} Y\right)$

$\sup (\mathrm{X})$.

Rule for Lift

The lift rule can be generated as a proportion of the above found support value with that predictable if 'X'\&'Y' where autonomous.

$$
\text { Lift }(X=>Y)=\sup \left(X U^{\prime} Y\right)
$$$$
\sup (\mathrm{X}) * \sup (\mathrm{Y})
$$

Rule for conviction can be described as $\operatorname{cnv}(\mathrm{X}=>\mathrm{Y})=$ $1--\sup (\mathrm{Y})$

$$
1--\operatorname{con}(X=>Y)
$$

Power Factor Rule

This particular rule is a demonstrating how powerful a rule is and the substance are related to another items with regard to constructive association-ship. The relationship may be represented by the following expression.

$$
\operatorname{pfr}(X=>Y)=\sup \left(X U^{\prime} Y\right) * \sup \left(X U^{\prime} Y\right)
$$$$
\sup (\mathrm{X})
$$

\section{Association Rule Application Method}

A rule for association is mainly implied to assure an end-user specific least amount of support value and an end-user specific least amount of confidence value in parallel way. Those rules for association generation are generated by two different tasks:

- A lowest amount of support value[12] threshold needs to be prepared in searching all numerous genes in genetic big data.

- A least amount of confidence value[12] restriction can be functioned in the recurrent genes to formulate the association rules.

\section{Sequence Pruning}

- Programmed DNA sequences are creating deprived class examines, especially close to sequence introduction site, also the closing stages of extended gene elements run. The genes from genetic documents usually has vector element sequences more often, The poly tails and additional not linked elements are the common occurrences noticed in gene sequence analysis. Amplified exons are usually bordered by introns and primer sequences. If these gene sequences are not altered by trimming, any one of these artifacts will amend your progression assembly and downstream sequence study. Sequencer tends to provide easy-to-use but influential tools that help to alter and prune reduced quality and indefinite data.

- TrimEnds tends to remove deceptive data from the split ending of gene sequencing remains.

- Trim Vector tends to remove sequence-specific data altering the ends of the required gene sequence element.

- Trim to Reference removes the edge of gene sequences that expand beyond an assembled orientation in a gene sequences. 
Correlation clustering- Objective:

- Unlimited number of clusters (No limitation on total numbers)

- Unlimited sizes of clusters (No specification of sizes)

Variants- Correlation based Clustering:

- CBC-Over lapping

- CBC-Chromatic

- CBC-Online

- CBCBipartite

- CBC-Aggregation

Table 4.1 Basic Variants of Correlation Based Clustering

\begin{tabular}{|l|l|l|}
\hline $\begin{array}{l}\text { Clustering } \\
\text { Constraint }\end{array}$ & \multicolumn{1}{|c|}{ CBC } & Overlapping- CBC \\
\hline $\begin{array}{l}\text { Objects as } \\
\text { set element }\end{array}$ & $\begin{array}{l}\mathrm{V}^{\prime}=\quad\{\mathrm{v} 1, \\
\mathrm{v} 2, \ldots \ldots, \mathrm{vn}\}\end{array}$ & $\begin{array}{l}\mathrm{V}^{\prime}=\quad\{\mathrm{v} 1, \quad \mathrm{v} 2, \ldots \ldots, \\
\mathrm{vn}\}\end{array}$ \\
\hline $\begin{array}{l}\text { Similarity } \\
\text { Function }\end{array}$ & $\mathrm{s}: \mathrm{V}^{\prime} \times \mathrm{V}, \rightarrow[0,1]$ & $\mathrm{H}: 2 \mathrm{~L} \times 2 \mathrm{~L} \rightarrow[0,1]$ \\
\hline $\begin{array}{l}\text { Labeling } \\
\text { Function }\end{array}$ & $1: \mathrm{V} \rightarrow \mathrm{L}$ & $1: \mathrm{V} \rightarrow 2 \mathrm{~L} \backslash\{0\}$ \\
\hline
\end{tabular}

\section{Correlation Based Clustering:}

To make the effective functions of CBC- clustering[13] functions are strongly associated with acknowledged distinct effective techniques. This study have projected a statistic based scrutiny of gene sequence models, which allow CBC task with regard to clustering task to approximate essential amount of genetic cluster elements. The above study considers purposes of consistent priority to every potential characteristic in spite of the total no of cluster.

The process of cluster the elevated dimension in gene sequence can analyze the clusters of genomic information with a few clusters to many thousands of dimensions. CBC- clusters is an attribute to dissimilar functions that correlated between different gene characters, attributes in elevated dimensioned samples are implicit to be present guided by rules for relating the process in cluster. This gene association can be diverse in dissimilar gene clustered elements, so a universal de-correlation may not decrease to customary clustering (un-associated). Correlations among subsets of gene sequences result in different spatial shapes of gene clusters being formed. Here, comparisons of gene group characteristics are described as obtaining to clarify the confined correlated prototypes. With this context, the term correlation based clustering has been introduced simultaneously with the context as mentioned. Diverse terms of $\mathrm{CBC}$ are discussed. Correlation based clustering is also found to be closely related to biclustering. The objective of biclustering process to recognize genes it split the association in several number of correlated distinctiveness that are association between the typical every separate cluster. Some of derivations and algorithms as followed:
Correlation based clustering to Overlapping Correlation Clustering:

Overlapping Clusters are very natural and slightly different from the correlation based clustering. It has better performance towards protein sequence analysis.

Table 4.4 Correlation Based Clustering Vs Overlapping Correlation Clustering

Clustering Constraint CBCOverlapping- CBC

Objects as set element $V^{\prime}=\{\mathrm{v} 1, \mathrm{v} 2, \ldots \ldots ., \mathrm{vn}\} \mathrm{V}^{\prime}=\{\mathrm{v} 1$, v2, ....., vn $\}$

Similarity Function s: V' x V, $[0,1] \quad \mathrm{H}: 2 \mathrm{~L}$ x $2 \mathrm{~L} \cdot$ $[0,1]$

Labeling Function $1: \mathrm{V} \cdot \mathrm{L} 1: \mathrm{V} \cdot 2 \mathrm{~L} \backslash\{0\}$

Clustering Function

$$
\mathrm{CCC}(\mathrm{L})=\sum_{(\mathrm{U}, \mathrm{V}) € \mathrm{Vx} \mathrm{V}}|\mathrm{s}(\mathrm{u}, \mathrm{v})-\mathrm{I}(1(\mathrm{U})=1(\mathrm{~V}))|
$$

$$
\begin{aligned}
& \text { Overlapping Clustering Function } \\
& \operatorname{COCC}(\mathrm{l})=\sum_{(\mathrm{U}, \mathrm{V})} € \mathrm{~V} \mathrm{x} \mathrm{V}(\mathrm{u}, \mathrm{v})-\mathrm{H}(\mathrm{l}(\mathrm{U}), \mathrm{l}(\mathrm{V})) \mid
\end{aligned}
$$

Overlapping Correlation Clustering Variants (r, H, p):

Values taken from Similarity function $\mathrm{s} \quad[0,1]$ $\mathrm{r}=\mathrm{f}$

Values taken from Similarity function $\mathrm{s} \quad\{0,1\}$ $\mathrm{r}=\mathrm{b}$

Jaccard coefficient of Similarity function $\mathrm{H} \quad \mathrm{H}=$ $\mathrm{J}$

The intersection indicator of similarity function $H \quad H=$ I

Maximum no of labels per obj as constraint $\mid 1(\mathrm{v})<\mathrm{p}, \mathrm{V}$ $\mathrm{v} € \mathrm{~V}$

Special cases: $\quad \mathrm{p}=1 \quad$ normal correlation clustering $\mathrm{p}=\mathrm{k}$ where $|\mathrm{L}|=\mathrm{k}$ no constraint

\section{EXPERIMENTAL SETUP}

Keep The experimental implementation is developed by the system using Java Virtual Machine (NET BEANS 8.1) and genomic data storage in a relational database (MS SQL 12.0). The experimental process started with a set of gene sequencing objects. The gene sequencing objects were stored in different clones of objects. The Java system generates information from gene sequencing objects and supplies data information associate to the singular phase of the sub sequencing task using an boundary for given $\mathrm{i} / \mathrm{p}$. Sub-sequences were produced and afterward it integrated, interpreted with reference to find the open or distributed genetic big data. With this, easy correlation clustering tasks toward clustering genetic elements to decide the supposed poly-morph data points were executed.

Different methods for correlation based clustering are available the relationship to different types of clusters are established using definite patterns. This research over indenture during the evolution of genetic discretization model with apposite protection surfaces in gene distributed database which was called Splice Dataset. 
considered to as donors.

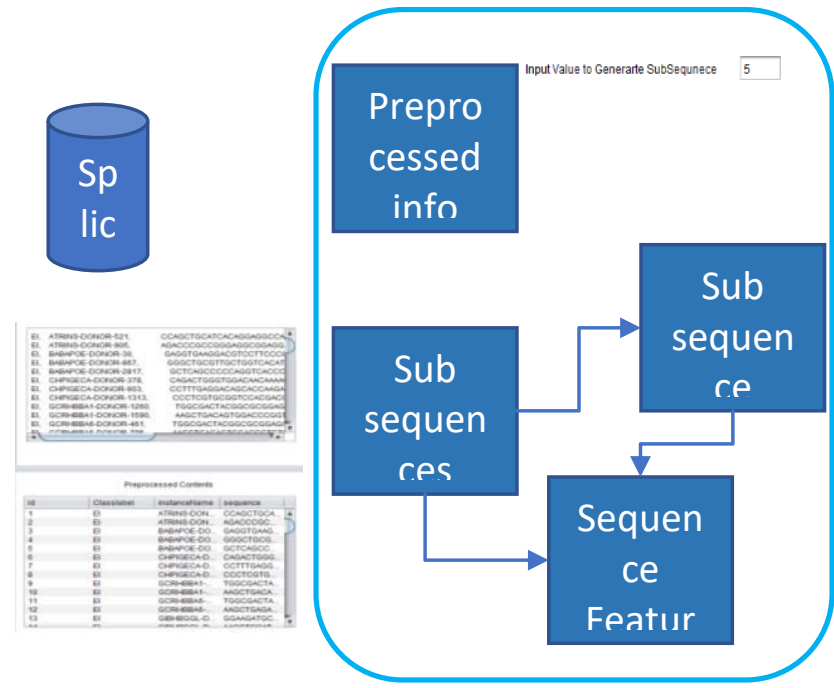

Figure 5.1 Java Model of Proposed Study

The JVM and a relational database combination provide the improvement in performance. This provides a simple system to preserve and scale in an effective database. In order to implement there is no need of additional expert knowledge in Java and SQL. Only basic familiarity towards JVM, MSSQL, also an admin of an object database is needed. Every beginner programmer can also revise and modify the model product.

The classification of each DNA gene element may be refer to the metaphors of mainly homologous DNA gene elements are in present with open and distributed database objects. The classification was done for DNA gene to sequence as of where a gene sequence is resulted or not from the distributed dataset. The homo-logos genetic elements from another type will offer descriptions to element's performance task. The clarification models have a benefit which model has completely automotive system. The descriptions may be effortlessly simplified and extracted focus gene element metaphors from novel explorations.

In this experiment, Total of thousand instance elements erratically chosen from the absolute set contains 3299 (In phase-1, 000 used). Splice data junctions are considered as data points on a DNA gene sequence. In the data points of DNA gene expressions 'super-fluous' gene sequences are separated through the task of mutation-protein design of DNA advanced organism sequence generation. Splice dataset has an issue that is to distinguish in the prearranged genetic elements, and also limitation of introns, exons are: part of gene elements preserved following 'splice'-ing process \& other part of genetic elements are to be generated in 'spliced' -out.

This defined problem definition has two more sub processes: to recognize coded gene/non coded gene margins, and to discover noncoded/coded margins. In genetic region, noncoded to coded-IE margins were regarded as acceptors while coded to noncoded genes were
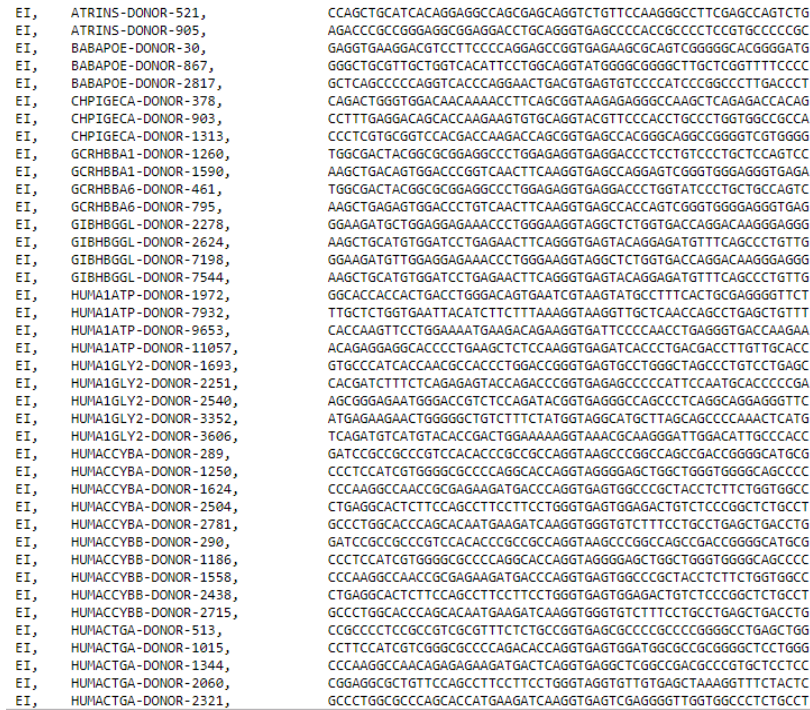

Figure 5.2 Gene Sequence with DNA Donor Id

\section{RESULTS AND DISCUSSION}

The basic gene discretization module observes the results towards the routine of projected technique with the total no. rules applied, precisions, re-call, correctness and time taken for executing. The following generated Table 6.1 demonstrates the different performance evaluators of splice dataset, Table 6.2 depicts performance of different classifier- clustering performance in splice, Table 6.3 for classification correctness, Table 6.4 with detailed cluster performance listed out the various detailed test results about the proposed work. Table 6.5 covers the performance evaluators compared with genetic data and application algorithm.

Performance Evaluators:

The analysis process of the performance of based on some parameters mentioned in the literature study. The proposed work results compared the total no.rules applied, precisions, re-call, correctness and time taken for executing.

The Table 6.1 contains performance evaluators such as accuracy, execution time, etc. These performance evaluators listed with genetic data and application algorithm the table.

Table 6.1 Performance Evaluators

\begin{tabular}{|l|l|l|l|}
\hline $\begin{array}{c}\text { Gene } \\
\text { Data }\end{array}$ & $\begin{array}{l}\text { Performance } \\
\text { Evaluators }\end{array}$ & $\begin{array}{l}\text { Application } \\
\text { Algorithm }\end{array}$ & Unit \\
\hline \multirow{4}{*}{$\begin{array}{c}\text { Splice } \\
\text { Dataset }\end{array}$} & Number of rules & $\begin{array}{l}\text { Clustering and } \\
\text { Classification }\end{array}$ & Value(No) \\
\cline { 2 - 4 } & Precision & $\begin{array}{l}\text { Clustering and } \\
\text { Classification }\end{array}$ & Value(No) \\
\cline { 2 - 4 } & Recall & $\begin{array}{l}\text { Clustering and } \\
\text { Classification }\end{array}$ & Value(No) \\
\cline { 2 - 4 } & Accuracy & $\begin{array}{l}\text { Clustering and } \\
\text { Classification }\end{array}$ & Percentage \\
\cline { 2 - 5 } & Execution time & $\begin{array}{l}\text { Clustering and } \\
\text { Classification }\end{array}$ & Seconds \\
\hline
\end{tabular}




\begin{tabular}{|l|l|r|r|l|l|l|l|}
\hline $\begin{array}{l}\text { Gene } \\
\text { 'N' }\end{array}$ & $\begin{array}{l}\text { UFS } \\
\text { FS }\end{array}$ & $\begin{array}{l}\text { UFR } \\
\text { FS }\end{array}$ & $\begin{array}{l}\text { FRM } \\
\text { IM }\end{array}$ & $\begin{array}{l}\text { Al } \\
\text { g1 }\end{array}$ & $\begin{array}{l}\text { C } \\
\text { FS }\end{array}$ & $\begin{array}{l}\text { UFR } \\
\text { DR }\end{array}$ & $\begin{array}{l}\text { CB } \\
\text { C }\end{array}$ \\
\hline 10 & 65 & 75 & 75 & 75 & 75 & 70 & 79 \\
\hline 20 & 82 & 95 & 92 & 84 & 78 & 75 & 95 \\
\hline 30 & 72 & 83 & 92 & 85 & 78 & 75 & 95 \\
\hline 40 & 72 & 90 & 90 & 85 & 87 & 72 & 92 \\
\hline 50 & 72 & 90 & 90 & 85 & 85 & 75 & 92 \\
\hline
\end{tabular}

The table 6.2 contains following data elements are checking the performances of splice data using classifier ROC and accuracy in existing algorithms, In table 6.2 contains accuracy of CBC-MLRC, also table 6.3. Shows the classification correctness of CBC-MLGC in top ' $n$ ' genes, the table 6.4 compares the multi different class data accuracy for CBC-MLGC

Table 6.2 Accuracy and ROC of CBC-MLGC In Splice Dataset

\begin{tabular}{|c|l|l|l|}
\hline \multirow{4}{*}{$\begin{array}{c}\text { Splice } \\
\text { Dataset }\end{array}$} & Algorithms & Accuracy & ROC \\
\cline { 2 - 4 } & c-4.5 & 89.25 & 90.2 \\
\cline { 2 - 4 } & naïve Bayes & 91.6 & 92.5 \\
\cline { 2 - 4 } & SVM & 90.2 & 91.64 \\
\cline { 2 - 4 } & simple Cart & 89.54 & 90.35 \\
\cline { 2 - 4 } & K-NN & 90.62 & 91.54 \\
\cline { 2 - 4 } & Proposed(MLGC) & 92.87 & 93.12 \\
\hline
\end{tabular}

The above table 6.2 shows the proposed algorithm compared with various algorithms classifier-naïve Bayesian, Support Vector Machine, K-NN and simple cart. The above table depicts that the proposed classifier MLGC works better than the other classifiers. Proposed algorithm, Naïve Bayes and K-NN are the top performing algorithms in the classifier performance accuracy. Based on ROC, the top performing algorithms are proposed (MLGC), Naïve Bayes and SVM classifiers.

This comparative analysis performed exclusively for the splice gene sequence dataset.

\section{Table 6.3 CBC-MLGC Correctness for Top 'N' Genes}

The proposed algorithm handles classification task in DNA sequence gene dataset, the below representation depicts the algorithm's accurateness for Top ' $n$ ' genes in CBC-MLGC.

The below table, the accuracy is calculated for top ' $n$ ' number of genes, the ' $n$ ' genes incremented by 10 genes each and calculated with further accuracy. The algorithms compared for the genetic data(increased 10 genes) per sequence with existing techniques and planned technique (CBC-MLGC). The below table shows that proposed algorithm performed with better results in every aspects of gene sequence.

Clustering performance in Splice Dataset

The detailed performance of above mentioned dataset is demonstrated in the Table 4.8. The table has proposed algorithm compared with various algorithms classifier- naïve Bayesian, Support Vector Machine, K-NN and simple cart.. This table having the performance measures such as classification accuracy, ROC and execution time. The above table depicts that the proposed classifier MLGC works better than the other classifiers. Proposed algorithm, Naïve Bayes and K-NN are the top performing algorithms in the classifier performance accuracy. Based on ROC, the top performing algorithms are proposed (MLGC), Naïve Bayes and SVM classifiers. Based on comparative ranking accord to the execution time, the proposed algorithm performs better than other classifiers. This comparative analysis [14] performed exclusively for the splice gene sequence dataset[15].

Table 6.5 CBC-MLGC Multiple Class Classification Correctness

\begin{tabular}{|c|c|c|c|c|c|c|}
\hline $\begin{array}{l}\text { Multiple } \\
\text { class } \\
\text { Data }\end{array}$ & $\begin{array}{l}\text { Kerne } \\
\text { lPLS+ } \\
\text { KNN }\end{array}$ & $\begin{array}{l}\text { GA- } \\
\text { ESP }\end{array}$ & $\begin{array}{l}\text { TS } \\
\mathrm{P}\end{array}$ & $\begin{array}{l}\mathrm{M} \\
\mathrm{O} \\
\mathrm{E} \\
\mathrm{D} \\
\mathrm{A}\end{array}$ & K-TSP & $\begin{array}{l}\text { CBC- } \\
\text { MLGC }\end{array}$ \\
\hline SRBCT & 96 & 98 & 95 & $\begin{array}{l}95 \\
.6\end{array}$ & 99 & 98 \\
\hline Lung & 95 & 90 & $\begin{array}{l}83 . \\
6\end{array}$ & $\begin{array}{l}95 \\
.7\end{array}$ & 94 & 97 \\
\hline $\begin{array}{l}\text { Splice } \\
\text { Dataset }\end{array}$ & 95 & 95 & 96 & 96 & 95 & 96 \\
\hline Leukemia & 99 & 96.5 & $\begin{array}{l}97 . \\
1\end{array}$ & 99 & 97.1 & 99 \\
\hline
\end{tabular}

\begin{tabular}{|l|l|l|l|l|l|}
\hline \multicolumn{7}{|c|}{ Clustering performance in Splice Dataset } \\
\hline $\begin{array}{l}\text { Classifie } \\
\mathrm{r}\end{array}$ & $\begin{array}{l}\text { Correctly } \\
\text { Classifie } \\
\mathrm{d}\end{array}$ & $\begin{array}{l}\text { Wrongly } \\
\text { Classifie } \\
\mathrm{d}\end{array}$ & $\begin{array}{l}\text { Accuracy } \\
(\%)\end{array}$ & $\begin{array}{l}\text { ROC } \\
\text { Curve } \\
(\%)\end{array}$ & $\begin{array}{l}\text { Exec } \\
\text { ution } \\
\text { Time } \\
(\mathrm{Sec})\end{array}$ \\
\hline C4.5 & 89.25 & 10.75 & $89 \%$ & 90.2 & 0.04 \\
\hline $\begin{array}{l}\text { Naïve } \\
\text { Bayes }\end{array}$ & 91.6 & 8.4 & $91 \%$ & 9 & 0.03 \\
\hline SVM & 90.2 & 9.8 & $90 \%$ & 91.64 & 0.04 \\
\hline $\begin{array}{l}\text { Simple } \\
\text { Cart }\end{array}$ & 89.54 & 10.46 & $90 \%$ & 90.35 & 0.05 \\
\hline K-NN & 90.82 & 10.38 & $91 \%$ & 91.54 & 0.03 \\
\hline $\begin{array}{l}\text { Propose } \\
\text { d } \\
\text { (MLGC) }\end{array}$ & 92.87 & 7.13 & $93 \%$ & 93.12 & 0.02 \\
\hline
\end{tabular}

Table 6.4 Clustering Performance Comparison in Splice Dataset

Clustering performance in Splice Dataset

CBC-MLGC Performance with Multiple Dataset Correctness 


\section{CONCLUSION}

This research contains 2 different datasets the training dataset and testing dataset. The association rules were framed to identify mutation diabetic genes in selected splice data set. The sequence pruning was implemented, mean finding was done. Finally CBC and MLGC were applied for result classifications. The experimental process consists of 1000 samples selected at random from set of 3190 splice database. Clustering performance for splice dataset was evaluated using 6 different algorithms as tabulated. The significance of constraint correctness accuracy and ROC were taken for replication research study. The CBC MLRC algorithm produced many clusters repeatedly with correctness around $92.87 \%$ and ROC of 93.12\%.The classification accurateness was summarized in table 6.2 for $n$ genes. The proposed method produced improved classification accurateness with respect to increasing number of cluster groups. Comparison of classification accuracy of multi class datasets were tabulated in table 6.3. The efficiency of the proposed CBC MLRC was proven with an average above 96 with the least execution time. By using data mining technique the diversity of gene sequences has reduced considerably .The clustering technology has also helped to establish the sequences of extracted gene data. By comparing and filtering multi class gene cluster data a determined accuracy has been attained in gene sequence dataset .The association rules drafted for testing data with support and confidence calculations has found to be successful. The MLRC algorithm has produced accurate results with reduced execution time. Thus it has been concluded from the results that CBC MLRC method has the fastest execution algorithm with reduced cost and improved accuracy.

\section{ACKNOWLEDGEMENTS}

I would like to thank Dr. S.SHEEJA for her expert supervision. Her wise academic advice and ideas have played an extremely important role in the work presented in this research. Without her support, this work would not have been possible.

\section{REFERENCES}

[1] What are Introns and Exons? By Michael Greenwood, M.Sc.

https://www.news-medical.net/life-sciences/What-areintrons-and-exons.aspx

[2] Vijay Arputharaj J, Dr.S.SheejaCorrelation-based Clustering and the Modified Naïve-BayesianClassification for Gene-sequence data analysis, International Journal of Engineering \& Technology(UAE), Volume 7 (4) (2018), PP 52925299, 2018

[3] Hao Helen Zhang Et al, Gene selection using support vector machines with non-convex penalty, Bioinformatics, Volume 22, Issue 1, 1 January 2006, Pages 88-95,
[4] Support-vector machine, From Wikipedia, the free encyclopedia, https://en.wikipedia.org/wiki/Supportvector machine

[5] Sanz, H., Valim, C., Vegas, E. et al. SVM-RFE: selection and visualization of the most relevant features through non-linear kernels. BMC Bioinformatics 19, 432 (2018).

[6] Devi ArockiaVanitha C, DevarajD,Venkatesulu M, Gene Expression Data Classification using Support Vector Machine and Mutual Information-based Gene Selection, Procedia Computer Science Elsevier, Volume 47 ( 2015 ) PP 13 - 21, 2015

[7] John $H$ and Brian Oliver, Microarrays, deep sequencing and the true measure of the transcriptome, BMC Biol. 2011; Volume 9: 34, Published online 2011 May 31. doi: 10.1186/1741-7007-9-34

[8] An Introduction to Naïve Bayes Classifier, By Yang $\mathrm{S}$ https://towardsdatascience.com/introduction-tona\%C3\%AFve-bayes-classifier-fa59e3e24aaf

[9] Molecular Biology (Splice-junction Gene Sequences) Data Set, Machine Learning Repositary, https://archive.ics.uci.edu/ml/datasets/Molecular+Biol ogy+(Splice-junction+Gene+Sequences)

[10] Vijay Arputharaj J, Dr.S.Sheeja“'Correlation Based Clustering and the Modified Naïve Bayesian Classification for Gene sequence data analysis", International Journal of Computer Technology \& Applications, Vol 9(1), Jan-Feb 2018, PP 24-29.

[11]Rajak, Akash. (2008). Association rule miningApplications in various areas. International Conference on Data Management (2008) https://www.researchgate.net/publication/238525379_ Association_rule_mining_Applications_in_various_ar eas

[12] Agrawal, R.; Imieliński, T.; Swami, A. (1993). "Mining association rules between sets of items in large databases". Proceedings of the 1993 ACM SIGMOD international conference on Management of data - SIGMOD '93. p. 207, 1993.

[13] Fujiwara, Koichi \& Kano, Manabu \&Hasebe, Shinji. (2010). Development of correlation-based clustering method and its application to software sensing. Chemometrics and Intelligent Laboratory Systems. 101. 130-138. 10.1016/j.chemolab.2010.02.006.

[14] "An Analysis of Modified Naïve Bayesian Classification using Correlation based Clustering for Gene Sequence Data Analysis", International Journal of Engineering \& Technology(UAE), Volume 7, Issue 4.5, Aug-Sep 2018, PP 612-616

[15] Splice dataset https://www.cs.toronto.edu/ delve/data/splice/desc.ht $\underline{\mathrm{ml}}$ 


\section{AUTHORS PROFILE}

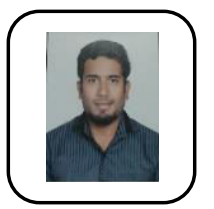

Dr. Vijay Arputharaj $\mathrm{J}$ is a Doctorate in Computer Science; He has also completed an integrated Post Graduate, Masters in Software Systems 2005-2010, Bharathiar University. A professional with 10 years of progressive leadership experience in lecturing. Currently he is having working experience in India, Ethiopia and Nigeria. He has served as Head of the Department of Software Engineering, 2016-2018 at Jijiga University, Ethiopia. Carried out additional responsibilities as Exam Cell Convener, 2012-2014 at VLB Janakiammal College, India by conducting centralized internal exams and preparing schedules. Has also undertaken training and development programs such as "Enhancing Teaching Skills" conducted by Wipro and SKASC at Coimbatore, August 2013. Followed by training on "Academic Performance Indicators" at Bharathiar University, January 2012.

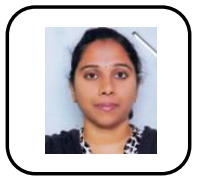

Ms. Pushpa Rega Ganesan, is currently working as a Lecturer in Department of Software Engineering, Institute of Technology, Jigjiga University, Ethiopia. She has completed her higher studies under Anna University, India. She has more than 5 years of experience in teaching field. She has extended her service in collaborative research projects and valuable supervision, guidance in under graduate engineering projects. She has taught different courses such as operating systems, human computer interaction, microprocessor, compiler design etc. She is actively participating in lecturing and research publication works. She is also interested in research areas of big data, gene mining, gene sequence data analysis etc.

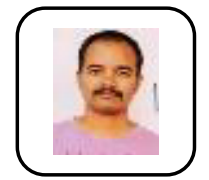

Mr. Ponsuresh Manoharan is currently working as a Senior Lecturer of Information Technology department in Institute of Technology, Jigjiga University, Ethiopia. Before joining Jigjiga University, he served in PSNA College of Engineering and Technology, India. He has completed his higher studies in Sathyabama University, Chennai. He has more than 11 years of experience and valuable publications in teaching and research field. He has extended his service in collaborative research projects, community services and valuable supervision of undergraduate student projects. He taught different courses like Java programming, Android Programming, Python Programming, Integrative programming to name a few. He is actively participating in lecturing and research publication works. $\mathrm{He}$ is also interested in research areas of Big Data, Gene Mining, Gene sequence and data analytics etc.

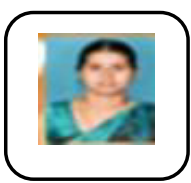

Ms. P.Supraja, is currently working as an Assistant professor in Department of Commerce, Hindusthan College of Arts and Science, Coimbatore, India. She has completed her higher studies under Bharathiar University, India. She has more than 10 years of experience in teaching field. Even though she is a commerce/management faculty, she has a good interest and knowledge in computer science researches also. She has completed valuable certifications in computer applications also. She also served as a System specialized faculty in VLB Janakiammal College of Arts and Science. She has interested in collaborative research projects. She has taught different courses such as Visual Basic, Visual Basic.Net, Database Management System, Modeling Languages etc. She is actively participating in lecturing and research publication works. Her contribution towards writing this research paper is appreciative. She is an active member in research, currently she is doing her doctorate in Bharathiar University, Coimbatore. 